\title{
Motion of a magnetic flow follower in two-phase flow - application to the study of airlift reactor hydrodynamics
}

\author{
Jaroslav Klein, ${ }^{1}$ Štefan Godó ${ }^{2}$ and José A Teixeira ${ }^{1 *}$ \\ ${ }^{1}$ Centro de Engenharia Biológica, Universidade do Minho, 4710-057 Braga, Portugal \\ ${ }^{2}$ Department of Chemical and Biochemical Engineering, Faculty of Chemical and Food Technology, Slovak University of Technology, \\ 81237 Bratislava, Slovakia
}

\begin{abstract}
A low-cost and simple magnetic particle tracer method was adapted to characterize the hydrodynamic behavior of an internal- and an external-loop airlift reactor (ALR). The residence time distribution of three magnetic particles differing in diameter $(5.5,11.0$ and $21.2 \mathrm{~mm})$ and with a density very close to that of water was measured in individual reactor sections. The measured data were analyzed and used to determine the velocity of the liquid phase. Validation of the experimental results for liquid velocity was done by means of the data obtained by an independent reference method. Furthermore, analysis of the differences found in the settling velocity of the particle in single-liquid and gas-liquid phases was carried out, using a simplified $3 D$ momentum transfer model. The model considering particle-bubble interaction forces resulting from changes in the liquid velocity field due to bubble motion was able to predict satisfactorily the increase in the particle settling velocity in the homogeneous bubbly regime. The effective drag coefficient in two-phase flow was found to be directly dependent on particle Reynolds number to the power of -2 but independent of gas flow-rate for all particle diameters studied. Based on the experimental and theoretical investigations, the valid exact formulation of the effective buoyancy force necessary for the calculation of the correct particle settling velocity in two-phase flow was done. In addition, recommendations concerning the use of flow-following particles in internal-loop ALRs for liquid velocity measurements are presented.
\end{abstract}

(C) 2006 Society of Chemical Industry

Keywords: multiphase flow; airlift reactors; hydrodynamics; momentum transfer; solid mechanics, flow follower

\section{NOTATION}

A Cross-sectional area, $\mathrm{m}^{2}$

$C_{\mathrm{D}} \quad$ Drag coefficient

$d \quad$ Diameter, $\mathrm{mm}$

$H \quad$ Vertical height, $\mathrm{m}$

$Q \quad$ Flow-rate, $\mathrm{m}^{3} \mathrm{~s}^{-1}$

$R e_{\mathrm{p}} \quad$ Reynolds number of particle $\left(=\rho_{\mathrm{L}} V_{\text {rel }} d_{\mathrm{p}} / \mu_{\mathrm{L}}\right)$

$R \quad$ Radius, $\mathrm{m}$

$U_{\mathrm{GRc}}$ Superficial gas velocity referred to the riser diameter (averaged at geometrical center of column), $\mathrm{m} \mathrm{s}^{-1}$

$U_{\mathrm{b}} \quad$ Bubble rise velocity, $\mathrm{m} \mathrm{s}^{-1}$

$U_{\mathrm{L}} \quad$ Liquid superficial velocity (referred to a reactor wall), $\mathrm{m} \mathrm{s}^{-1}$

$U_{\text {Lexp }}$ Liquid velocity measured by a magnetic flow follower, $\mathrm{m} \mathrm{s}^{-1}$

$U_{\text {Lref }}$ Liquid velocity measured by a reference method (orifice), $\mathrm{m} \mathrm{s}^{-1}$

$U_{\mathrm{p}} \quad$ Particle settling velocity in single-liquid phase, $\mathrm{m} \mathrm{s}^{-1}$

$V \quad$ Linear velocity, $\mathrm{m} \mathrm{s}^{-1}$

$V_{\mathrm{p}} \quad$ Particle velocity (referred to a reactor wall), $\mathrm{m}$ $\mathrm{s}^{-1}$
$V_{\text {rel }}$ Relative particle to liquid velocity in gas-liquid mixture, $\mathrm{m} \mathrm{s}^{-1}$

$v \quad$ Local liquid velocity, $\mathrm{m} \mathrm{s}^{-1}$

$v_{\mathrm{p}} \quad$ Local particle velocity, $\mathrm{m} \mathrm{s}^{-1}$

$V_{\text {rel }}$ Relative particle to liquid velocity in gas-liquid mixture, $\mathrm{m} \mathrm{s}^{-1}$

$x \quad$ Horizontal distance between bubble and particle, $\mathrm{m}$

$y \quad$ Vertical distance between bubble and particle, $\mathrm{m}$

$z \quad$ Horizontal distance between bubble and particle, $\mathrm{m}$

\section{Greek symbols}

$\varepsilon \quad$ Holdup

$\mu \quad$ Viscosity, $\mathrm{Pa} \mathrm{s}$

$\rho \quad$ Density, $\mathrm{kg} \mathrm{m}^{-3}$

$\eta_{\mathrm{UL}} \quad$ Deviation of $U_{\mathrm{L}}$ values determined by magnetic method from reference values, $\%$

\section{Subscripts \\ act Maximal active \\ b Bubble}

\footnotetext{
* Correspondence to: José A Teixeira, Centro de Engenharia Biológica, Universidade do Minho, 4710-057 Braga, Portugal E-mail: jateixeira@deb.uminho.pt

Contract/grant sponsor: Fundação para a Ciência e a Tecnologia (FCT); contract/grant number: SFRH/BPD/5607/2001 (Received 2 February 2005; revised version received 3 April 2006; accepted 21 April 2006)

Published online 2 October 2006; DOI: 10.1002/jctb.1604 
c Averaged at geometrical center of column (temperature and pressure)

C Circulation/column

cal Calculated using model

D Downcomer

dis Gas-liquid dispersion

DT Draft tube

ef Effective

exp Experimental

G Gas phase

L Liquid phase

p Particle

R Riser

ref Reference

$x \quad x$-Coordinate, horizontal direction

$y \quad y$-Coordinate, vertical direction

\section{INTRODUCTION}

In recent years, multiphase airlift reactors (ALRs) have been studied extensively as promising devices for two- and three-phase bio/chemical applications. Especially for three-phase biosystems, an ALR possesses attractive properties, such as efficient suspension of solids at low power input combined with low and homogeneously distributed shear stress. In airlift bioreactors with a well-defined circulation loop, the net liquid velocity is the major hydrodynamic parameter, which considerably affects all physical phenomena (mixing, mass and heat transfer, etc.). The development and verification of empirical correlations and semi/theoretical models describing the reactor hydrodynamics rely on experimental measurements. Despite an extensive number of velocity measuring methods applied to ALRs, ${ }^{1}$ most of these techniques are not suitable for use in fermentation processes, for several reasons [tagging of liquid elements with chemicals due to their interference with the strictly controlled composition of the fermentation medium and sterility problems, visual techniques such as laser Doppler anemometry (LDA) or particle image velocimetry (PIV) due to the opaqueness of the broth].

The applicability of any measuring technique to real biosystems calls for simple installation and non-toxic, aseptic and long-term stable usage. Moreover, any direct intervention inside the bioreactor is usually not allowed owing to its undesirable effect on the bioprocess operation. The use of small, inert, flow-following particles with non-invasive detection of their movement is a promising method. Detection techniques for opaque media include the use of radioactive counters, ${ }^{2,3}$ inductive coils ${ }^{4,5}$ and radiowave detectors. ${ }^{6,7}$ The cheapest and simplest method is the use of a magnetic-detector coil technique. A system of two inductive coils fixed at the outer wall of an inner draft tube in an internalloop ALR was used by Kikuchi et al. ${ }^{4,8}$ to measure the particle velocity in the riser and downcomer reactor sections. However, in such a configuration, this device is not suitable for use in bioprocesses owing to bioreactor sterility and biomass adhesion problems. The technique using a magnetic particle and inductive coils placed outside the reactor at the outer wall of the column was described by Klein et al. ${ }^{9}$ The technique was then successfully tested to measure the particle and liquid velocities in both main sections of an internal-loop airlift bioreactor during gluconic acid production ${ }^{10}$ and continuous alcoholic fermentation of cheese whey. ${ }^{11}$

A substantial problem related to the application of flow followers is the determination of the relative particle to liquid velocity. There is a major discrepancy in the literature regarding the definition of the effective buoyancy force (is it based on liquid or bulk density?), which is coupled with the complexity of quantification of particle-bubble (G-L) or particle-particle $(\mathrm{G}-\mathrm{L}-\mathrm{S})$ interactions. Several papers claim that the buoyancy is based on the hydrostatic pressure and therefore it is related to the bulk (suspension) density. ${ }^{12-14} \mathrm{Schmidt}^{12}$ determined the effective buoyancy force based on the density of the gas-liquid dispersion and obtained good agreement between the flow-follower method and results of the pulse $\mathrm{pH}$ technique. Others ${ }^{15-17}$ claim that the buoyancy force is the proper Archimedes lift force; therefore, the tracer particle experiences the liquid density. As suggested by Fan et al., ${ }^{16}$ the buoyancy force based on the liquid density should be called the Archimedes buoyancy, and that based on the bulk density (dispersion or suspension) the apparent buoyancy.

However, there is circumstantial evidence supporting the use of a specific criterion to decide whether the particle experiences the bulk density or the liquid density. Grbavcic and Vukovic ${ }^{17}$ studied the settling of a tracer particle in a fluidized bed and found that the effective buoyancy was related to the suspension density for a particle with a diameter much larger than that of the surrounding particles (more than five times), whereas in the case of approximately equal diameters the tracer particle experiences liquid density. The same phenomenon but in a bubble-liquid dispersion was observed by Middleton. ${ }^{18}$ It seems that a critical diameter of the tracer particle with respect to the diameter of surrounding particles or bubbles exists, influencing the proper formulation of the effective buoyancy (apparent or Archimedes buoyancy).

There is also a great divergence in the literature regarding the findings on how surrounding bubbles or particles affect the settling velocity of the tracer particle. The relative particle to liquid velocity in two- or three-phase flow $\left(V_{\text {rel }}\right)$ was found to be lower than the settling velocity in the single-liquid phase $\left(U_{\mathrm{p}}\right)^{8,19-21}$ The decrease in the settling velocity in a gas-liquid medium was caused by an increase in gas flow-rate depending on the particle size. It was found that bubbles did not affect the settling velocity of a particle if its diameter was around $0.7 \mathrm{~mm}^{20}$ or less than $0.46 \mathrm{~mm} .{ }^{19}$ In contrast, several papers ${ }^{22-25}$ have reported higher values of the settling velocity in 
two/three-phase flow than in a single-liquid phase. These discrepancies are most probably associated with differences in particle properties employed in the various studies.

The main task of the present work was to compare the experimental results obtained by the low-cost magnetic particle-tracer method with an independent reference method. Further, an analysis of the differences obtained was carried out, using a simplified momentum transfer model. The experimental and theoretical investigation resulted in the valid formulation of the effective buoyancy force necessary for the calculation of the correct particle settling velocity in two-phase flow.

\section{EXPERIMENTAL}

\section{Reference measurements in external-loop ALR}

The system used for reference measurements was a $36-\mathrm{dm}^{3}$ external-loop ALR with a total liquid height of $1.6 \mathrm{~m}$. Both riser and downcomer sections with a diameter of $0.1 \mathrm{~m}$ were connected by horizontal junctions of the same diameter at a distance of $0.6 \mathrm{~m}$. An enlarged head zone (with a diameter of $0.16 \mathrm{~m}$ ) at the top of the riser tube was used as a gas-liquid separator. A sintered-glass plate of $0.075 \mathrm{~m}$ diameter (pore size $150-200 \mu \mathrm{m}$ ) was used as a gas distributor. A calibrated orifice device of $0.06 \mathrm{~m}$ diameter, installed in the bottom junction tube, was used to measure the liquid flow-rate. The orifice flow meter was calibrated outside the reactor by means of a simple method of measurement of the time needed to fill a vessel with a certain accurate volume. The experimental error of the calibration procedure was up to $1 \%$ on average. Water and air were used as liquid and gas phases, respectively.

\section{Measurements in an internal-loop ALR}

A $50-\mathrm{dm}^{3}$ internal-loop ALR with an enlarged degassing zone was used for measurements of liquid velocity in the riser and downcomer zones. The reactor consisted of an outer column (diameter $0.142 \mathrm{~m}$, height $2 \mathrm{~m}$ ) equipped with an enlarged head zone (diameter $0.442 \mathrm{~m}$, height $0.35 \mathrm{~m}$ ) and a draught tube. Various reactor configurations with different diameters and lengths of the inner draft tube were used to test the accuracy of the magnetic particle-tracer technique. The reactor with all important dimensions and the equipment set-up can be seen in a previous paper. ${ }^{26}$

\section{Velocity measurements}

The magnetic particle-tracer technique was used to measure the residence time of the tracer particle in all individual reactor sections (riser, downcomer, separator). The measuring system utilizes two inductive coils, which are fixed around the outer wall of the column at a certain vertical distance. The coils oscillating at close, adjustable basic frequencies by means of oscillators detect the transition of the tracer particle with a high relative magnetic permeability (around 8000).
The two coils are coupled together by a magnetic bond in a quasi 'unstable state', in which coils try to adjust themselves to each other's frequency. The magnetic tracer particle passing through one of the coils induces a change in differential frequency, which is registered by an $\mathrm{A} / \mathrm{D}$ converter attached to a personal computer. For data processing, a special computer program was developed. A more detailed description of the equipment can be found in a previous paper. ${ }^{9}$

In the case of the internal-loop ALR, the coils fixed at the outer column were used to measure the particle residence time in both the riser and downcomer sections simultaneously. In practice, it is necessary to be able to distinguish whether a peak in the measured signal is induced by the particle passing through the inner riser or the annulus downcomer sections. In a previous study, ${ }^{9}$ it was found that the height of the peak is a function of the radial position of the particle. Thus, according to the peak height, the reactor section (draft tube or annulus) where the particle was situated could be easily identified. This was essential for correct peak identification during data processing. In the case of the external-loop reactor, the measurements were made separately in the riser and downcomer under identical operating conditions (gas flow-rate, temperature, etc.).

The magnetic particle-tracer technique permits the simple, fast and autonomous acquisition of a large amount of experimental data. In the present work, around 100 values were obtained for every experimental point when a dynamic steady state of the system was reached. More advantages are obtained by the use of two coupled coils as no correction for the thermal instability of oscillators is needed and any change of the medium composition affects both coils but not the frequency difference.

Three spherical particles with diameters of 5.5, 11.0 and $21.2 \mathrm{~mm}$ were prepared. The density of the magnetic tracer particle was adjusted to the density of the liquid phase as much as possible and a waterproof and bubble non-adherent elastomeric paint (gumelastic) was applied to the surface of the particle. The particle settling velocity in the single-liquid phase $\left(U_{\mathrm{p}}\right)$ was determined experimentally as the terminal settling velocity of one particle in a wide stagnantliquid column at a constant temperature of $20^{\circ} \mathrm{C}$ (the values are shown in Fig. 3 at zero gas flow-rate). Important properties of the magnetic particles used are listed in Table 1.

Table 1. Important properties of magnetic particles used in experiments: the settling velocity in a single-liquid phase was determined in tap water at a room temperature $\left(20.0^{\circ} \mathrm{C}\right)$

\begin{tabular}{lccr}
\hline Parameter & $\begin{array}{c}\text { Small } \\
\text { particle 1 }\end{array}$ & $\begin{array}{c}\text { Medium } \\
\text { particle 2 }\end{array}$ & $\begin{array}{c}\text { Large } \\
\text { particle 3 }\end{array}$ \\
\hline Diameter, $d_{\mathrm{p}}(\mathrm{mm})$ & 5.5 & 11.0 & 21.2 \\
Density, $\rho_{\mathrm{p}}\left(\mathrm{kg} \mathrm{m}^{-3}\right)$ & 998.1 & 998.7 & 1002.2 \\
Velocity, $U_{\mathrm{p}} \times 10^{2}\left(\mathrm{~m} \mathrm{~s}^{-1}\right)$ & 0.09 & 0.75 & 4.96 \\
\hline
\end{tabular}




\section{Gas holdup}

The gas holdup was determined by the standard manometric method in both external- and internalloop ALRs. Inverse manometric tubes were used for the measurement of pressure differences between two points in the riser and the downcomer of the ALR. The positions of the measuring points were properly chosen in order to avoid the effect of liquid acceleration at the bottom and the top of the draught tube. ${ }^{27}$ Then, the average overall gas holdups, $\varepsilon_{\mathrm{GR}}$ and $\varepsilon_{\mathrm{GD}}$, were determined with experimental error between 1 and $3.5 \%$.

\section{RESULTS AND DISCUSSION Reference measurements in an external-loop ALR}

The values of gas holdup in the riser $\left(\varepsilon_{\mathrm{GR}}\right)$ and superficial liquid velocity [ $U_{\mathrm{L}}$, see Eqns (1) and (2)] were used to identify two-phase flow regimes in the riser of the ALR (see Fig. 1). The gas holdup in the downcomer was found to be negligible in all experiments. The gas holdup curve has a logarithmic shape; however, two break points on the curve can be identified visually. They correspond to two-phase flow regime transitions. Similar breaks on the riser gas holdup curves were observed, e.g. by Merchuk et al. ${ }^{28}$ using a sintered-glass distributor with a pore size of $120 \mu \mathrm{m}$. For low gas superficial gas velocity, $\left(U_{\mathrm{GRc}}\right.$ up to $0.035 \mathrm{~m} \mathrm{~s}^{-1}$ ), homogeneous bubbly flow was observed with small bubbles (typically with a diameter around $3 \mathrm{~mm}$ ). There is a clear linear dependence of the gas holdup on $U_{\mathrm{GRc}}$ indicating that the upwardmoving bubbles were not affecting each other. At higher air flow-rates, the gas holdup curve is no longer linear, demonstrating the existence of the transition regime. At $U_{\mathrm{GRc}}$ values higher than $0.065 \mathrm{~m} \mathrm{~s}^{-1}$, heterogeneous churn-turbulent flow appears. Larger bubbles with an equilibrium diameter around $6 \mathrm{~mm}$ are formed as a consequence of coalescence events, as reported, for instance, by Heijnen and van't Riet. ${ }^{29}$

First, it was assumed that the tracer particle falls in the gas-liquid dispersion with a settling velocity equal to that in the single-liquid phase, $U_{\mathrm{p}}$. Then, the superficial liquid velocity in the corresponding reactor section $\left(U_{\mathrm{L}}\right)$ can be calculated from the experimental values of the particle velocity $\left(V_{\mathrm{p}}\right)$ as follows:

$$
\begin{aligned}
& U_{\mathrm{LR}}=\left(V_{\mathrm{pR}}+U_{\mathrm{p}}\right)\left(1-\varepsilon_{\mathrm{GR}}\right) \\
& U_{\mathrm{LD}}=\left(V_{\mathrm{pD}}-U_{\mathrm{p}}\right)
\end{aligned}
$$

These experimental values of superficial liquid velocity $\left(U_{\text {Lexp }}\right)$ were then compared with the reference values obtained by the standardized orifice device $\left(U_{\text {Lref }}\right)$. The deviation of $U_{\mathrm{L}}$ determined by the magnetic particle-tracer method is depicted in Fig. 2. The observed differences clearly demonstrate the existence of differences in settling velocity in singleand two-phase flows. Comparing Figs 1 and 2, one can

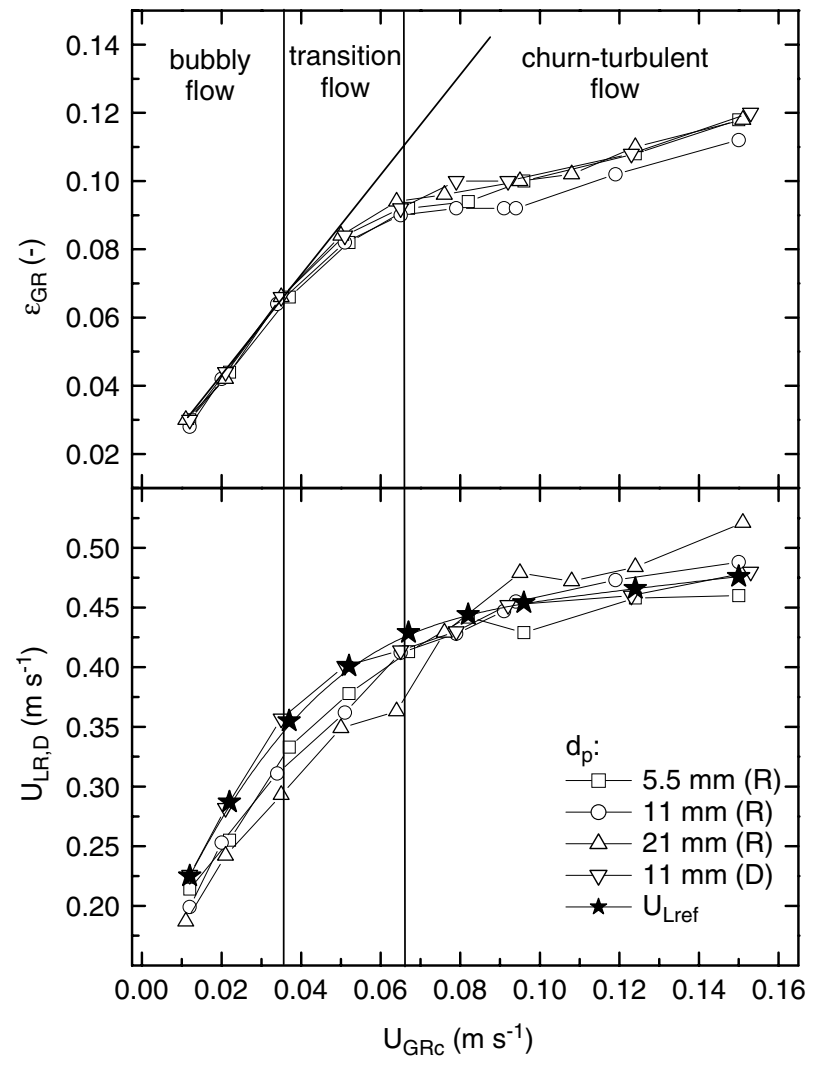

Figure 1. Gas holdup in the riser $\left(\varepsilon_{\mathrm{GR}}\right)$ and superficial liquid velocity $\left(U_{\mathrm{L}}\right)$ calculated from the particle velocity $\left(V_{\mathrm{p}}\right)$ using the values of velocity $U_{\mathrm{p}}$ versus the superficial gas velocity $\left(U_{\mathrm{GRc}}\right)$ for three different particle diameters $d_{p}$. Reference values of liquid velocity ( $\left.U_{\text {Lref }}\right)$ are also depicted for comparison. Letters $\mathrm{R}$ and $\mathrm{D}$ indicate the values of $U_{\mathrm{L}}$ in the riser and downcomer, respectively.



Figure 2. Percentage deviation of the superficial liquid velocity determined by the magnetic particle-tracer method corrected for the single-phase settling velocity, $U_{p}$ [see Eqns (1) and (2)], compared with the experimental values of the liquid velocity from reference measurements with the standard orifice in an external-loop ALR. Particles with different diameters $d_{\mathrm{p}}$ were used. Letters $R$ and $D$ indicate the values of $U_{\mathrm{L}}$ in the riser and downcomer, respectively. The vertical lines indicate transitions between flow regimes.

see that the sign and the magnitude of the deviation $\eta_{\mathrm{UL}}$ change with variation of the two-phase flow regime in the riser zone. The relative particle to liquid velocity 
in the gas-liquid dispersion $\left(V_{\text {rel }}\right)$ was then determined using experimental values of $U_{\text {Lref }}$ obtained by using the orifice flowmeter as follows:

$$
\begin{aligned}
V_{\text {Rrel }} & =\frac{U_{\text {LRref }}}{\left(1-\varepsilon_{\mathrm{GR}}\right)}-V_{\mathrm{pR}} \\
V_{\text {Drel }} & =\left(V_{\mathrm{pD}}-U_{\mathrm{LDref}}\right)
\end{aligned}
$$

In the homogeneous bubbly and transition flow regimes, the relative particle to liquid velocity $\left(V_{\text {rel }}\right)$ was higher than the settling velocity in a singleliquid phase (see Fig. 3). However, as bigger bubbles appeared in the churn-turbulent regime, $V_{\text {rel }}$ became smaller than $U_{\mathrm{p}}$. There was one exception in the case of the smallest tracer particle $\left(d_{\mathrm{p}}=5.5 \mathrm{~mm}\right)$, in which $V_{\text {rel }}$ was higher than $U_{\mathrm{p}}$ in all flow regimes. These facts indicate that diverse particle-bubble interaction mechanisms played a key role in different gas-liquid flow regimes. In the bubbly regime, the particle settling was probably accelerated by the liquid flowing down around rising bubbles and traveled outside a fastermoving liquid in bubble wakes. This resulted in a higher relative particle to liquid velocity, $V_{\text {rel }}$. In the transition and churn-turbulent regimes, the lumped hindering effect of another transport mechanism ('lifting' the particle by the bubble via its liquid layer, a larger contraction of cross-sectional area for particle movement) began to play an important role that resulted in $V_{\text {rel }}$ being approximately equal to or lower than $U_{\mathrm{p}}$. These findings are in an agreement with the results of visual and power spectral analysis by Kikuchi et al. ${ }^{30}$ They observed in bubbly flow that the bubbles exerted a wobbling motion, avoiding any encounters with the tracer particle, whereas in the churn-turbulent flow, larger coalescing bubbles moved upwards faster and more straightforward. In the churn-turbulent regime, a mechanism of direct particle-bubble collisions was evident, which hindered the particle settling. However, Kundakovic and Vunjak-Novakovic ${ }^{21}$ reported that the motion of particles with a density less than $1100 \mathrm{~kg} \mathrm{~m}^{-3}$ is not affected by the presence of bubbles and the particles closely follow liquid streamlines. In the present work, where particles with a density very close to that of water $\left(\rho_{\mathrm{H}_{2} \mathrm{O}}<\rho_{\mathrm{p}}<1003 \mathrm{~kg} \mathrm{~m}^{-3}\right)$ were used, it was demonstrated that the motion of even such particles could be affected by the presence of bubbles.

Regarding the measurements in the downcomer of the external ALR, the situation differed significantly from that in the riser section. The relative particle to liquid velocity, $V_{\text {rel }}$, was almost identical with $U_{\mathrm{p}}$; however, at high gas flow-rates the $V_{\text {rel }}$ values became slightly lower owing to a hindering effect of small bubbles (ca $1-2 \mathrm{~mm}$ ), which were entrained in a reduced amount in the downcomer and moved slowly downwards. Comparing the magnetic particle-tracer technique with the reference methods, the absolute average error of liquid velocity corrected for the $U_{\mathrm{p}}$ values was 1.9 and $5.9 \%$ in the downcomer and riser sections, respectively.

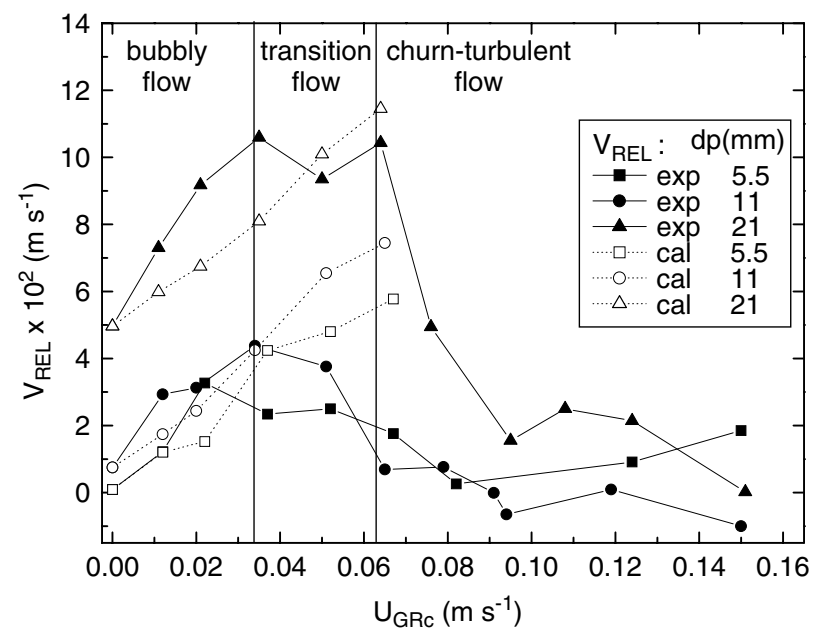

Figure 3. Experimental (solid symbols) and calculated (open symbols) values of relative particle to liquid velocity $V_{\text {rel }}$ as a function of superficial gas velocity in an external-loop ALR. $d_{\mathrm{b}}=3 \mathrm{~mm}$, $U_{\mathrm{b}}=0.25 \mathrm{~m} \mathrm{~s}^{-1}$, maximum active distance $x_{\mathrm{act}}=1 d_{\mathrm{b}}+0.5 d_{\mathrm{p}} . V_{\text {rel }}$ at $U_{\mathrm{GRc}}=0 \mathrm{~m} \mathrm{~s}^{-1}$ corresponds to $U_{\mathrm{p}}$.

Moreover, root mean square (RMS) values were calculated from the standard deviation of particle velocity, $V_{\mathrm{p}}$, giving an indication on the intensity of turbulence in the corresponding reactor section. The RMS values of $V_{\mathrm{p}}$ were found to be much lower in the downcomer than in the riser section owing to the absence of bubbles; however, at the highest gas flowrates they approached the values in the riser. This indicates that larger discrepancies in the residence time of the particle in the gas-liquid flow originate from particle-bubble interactions and the fluctuations in the fluid flow due to the rising bubbles inducing vortical and chaotic liquid motion.

\section{Effect of bubbles on particle motion}

To verify the present findings on particle-bubble interactions in two-phase flow, an extended 3D momentum transfer model originally suggested by Clift and Grace $^{31}$ was applied to describe the impact of the liquid velocity field created by bubble motion on the particle velocity in the concurrent gas-liquid flow. The model predicts the average vertical component of the settling velocity of a particle by solving Navier-Stokes equations applying several simplifications: homogeneous bubbly flow with the absence of direct bubble-bubble or particle-bubble interactions, uniform bubble size distribution, rigid bubble shape, initial position of particle in the vertical axis of the column. Thus, motion equations for components of the local particle velocity can be written in the following form, as suggested by Kundakovic and Vunjak-Novakovic: ${ }^{21}$

$$
\begin{aligned}
& v_{\mathrm{p} y}=U_{\mathrm{p}}+U_{\mathrm{b}} \frac{y^{2}-x^{2}}{\left(x^{2}+y^{2}\right)^{2}} R_{\mathrm{b}}^{2} \\
& v_{\mathrm{p} x}=U_{\mathrm{b}} \frac{2 x y}{\left(x^{2}+y^{2}\right)^{2}}{R_{\mathrm{b}}}^{2}
\end{aligned}
$$


where $x$ and $y$ represent the horizontal and vertical distances between the particle and the bubble. To extend the model mechanistically into $3 \mathrm{D}$, the effect of randomly distributed surrounding bubbles was accounted for by 'turning' the 2D Cartesian coordinate system for each bubble-particle pair. The maximum active distance (in $x, y$ coordinates) defining the area of influence of bubbles on the particle motion was estimated from the one-bubble model, which evaluates the impact of motion of one bubble on the particle settling. This parameter was found to be equivalent to $1 d_{\mathrm{b}}+0.5 d_{\mathrm{p}}$. The bubbles were assumed to have a rigid spherical shape with a diameter of $3 \mathrm{~mm}$ calculated using a correlation of Heijnen and van't Riet ${ }^{29}$ and confirmed by visual analysis. Several Monte Carlo simulations were performed (random initial distribution of bubbles for a given gas holdup) for each particle size. The relative particle to liquid velocity in Fig. 3 is the average over all the respective simulations.

The simulations showed that the momentum transfer model is able to predict satisfactorily the variations of the relative particle to liquid velocity in the vicinity of the bubbles at low values of gas flow-rate, where the homogeneous bubbly regime is assured (see Fig. 3). The calculated values of $V_{\text {rel }}$ increased with gas flow-rate; however, in several cases the model underestimated the velocity values. In the transition regime, where coalescence events cannot be neglected, the modeled values began to be higher than those obtained by measurements. This shows that the applied model is not suitable to estimate changes in relative particle to liquid velocity in the gas-liquid flow, where coalescence and bubble-bubble interactions become important. In these flow regimes (transition and churn-turbulent regimes), other particle-bubble interaction mechanisms (see the previous section) became significant, which slowed the settling of the tracer particles.

The modeled data on the relative particle to liquid velocity $\left(V_{\text {rel }}\right)$ were used to calculate the liquid velocity in the riser, $U_{\text {LRcal }}$ [applying $V_{\text {rel }}$ instead of $U_{\mathrm{p}}$ in Eqn (1)], where $V_{\mathrm{pR}}$ is the particle velocity measured by the particle-tracer technique. Comparison of liquid velocity determined by the particle-tracer method applying the settling velocity in a single phase $\left(U_{\text {LRexp }}\right)$, applying the relative particle to liquid velocity calculated from momentum transfer model ( $U_{\text {LRcal }}$ ) and the values determined by the reference method $\left(U_{\text {LRref }}\right)$ is shown in Fig. 4. The graph shows that the accuracy of liquid velocity determination by the particle-tracer method can be improved in the homogeneous regime using the $V_{\text {rel }}$ values calculated from the momentum transfer model [Eqns (5) and (6)].

The experimental and modeled data were also verified by a very simple experiment in a $0.07-\mathrm{m}$ bubble column with a liquid height of $0.5 \mathrm{~m}$. Particles with different diameters $(5-21.2 \mathrm{~mm})$ and a density

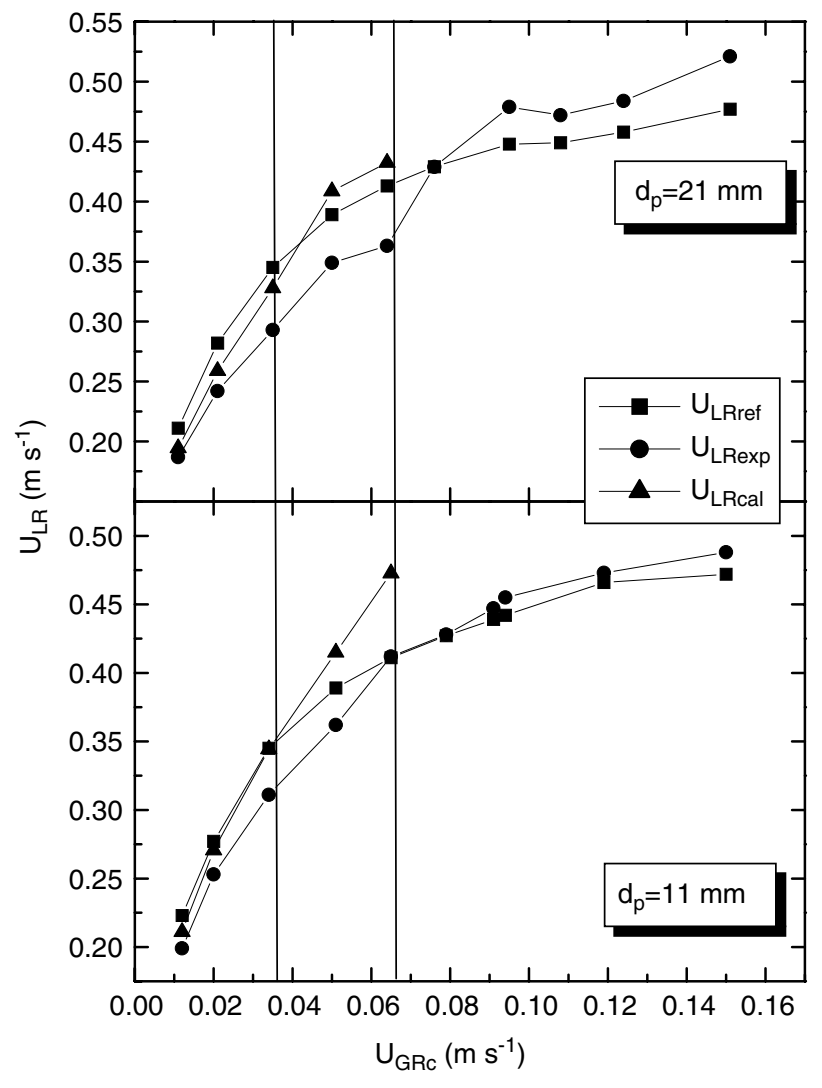

Figure 4. Comparison of liquid velocity determined by the particle-tracer method in an external-loop ALR applying the settling velocity in single phase $\left(U_{\text {LRexp }}\right)$, applying the relative particle to liquid velocity calculated from the momentum transfer model $\left(U_{\text {LRcal }}\right)$ and the values determined by the reference method $\left(U_{\mathrm{LRref}}\right)$ versus the superficial air velocity $U_{\mathrm{GRC}}$.

slightly lower than that of water were located close to the liquid surface in the column without aeration. As slight aeration was started, the particles began to fall rapidly and stayed in the bottom part of column. The same effect of bubbly flow on large $\left(d_{\mathrm{p}}=25 \mathrm{~mm}\right)$ and low-density $\left(\rho_{\mathrm{p}}=1030 \mathrm{~kg} \mathrm{~m}^{-3}\right)$ particles was observed by Mazumdar and Guthrie. ${ }^{24}$ They used more sophisticated equipment to determine the settling velocity of a particle in gas-liquid flow by regulating the liquid upflow rate in a bubble column. They found that a strong increase in the liquid flowrate was needed to keep a particle in an equilibrium vertical position when a gas was introduced into the column. This phenomenon was explained by a reduction in the effective drag coefficient (by $50 \%$ ); however, no explanation for this drag force attenuation was presented.

\section{Effect of Reynolds number on particle settling}

The effect of particle Reynolds number on its settling velocity was investigated using the force balance. Additional particle-bubble forces acting on the tagged particle in gas-liquid flow can be expressed via an effective drag coefficient determined as follows: ${ }^{21}$

$$
C_{\text {Def }}\left(\frac{\rho_{\mathrm{L}} V_{\text {rel }}^{2}}{2}\right)\left(\frac{\pi d_{\mathrm{p}}^{2}}{4}\right)=\left(\frac{\pi d_{\mathrm{p}}^{3}}{6}\right)\left(\rho_{\mathrm{p}}-\rho_{\mathrm{L}}\right) g
$$




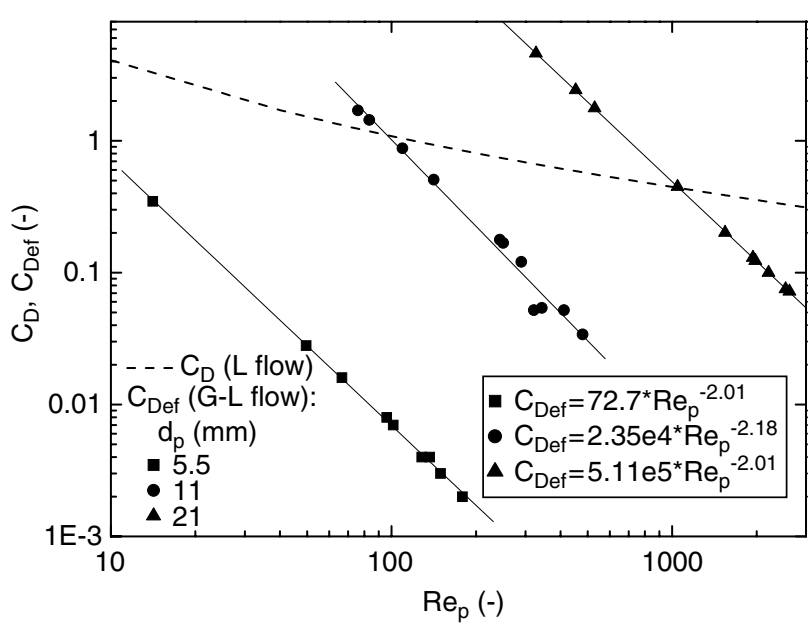

Figure 5. Effect of particle diameter and Reynolds number on effective drag coefficient $\left(C_{\text {Def }}\right)$ in gas-liquid flow in the riser zone of an external-loop ALR. The solid lines represent regression curves and the dashed line represents the drag coefficient in the liquid phase.

Comparison of the effective drag coefficient $\left(C_{\text {Def }}\right)$ in the gas-liquid phase and the drag coefficient in a single liquid phase $\left(C_{\mathrm{D}}\right)$ for different $d_{\mathrm{p}}$ values is depicted in Fig. 5. It is evident that $C_{\text {Def }}$ depends on the particle Reynolds number $\left(R e_{\mathrm{p}}\right)$ but it is independent of gas flow-rate (or gas holdup). The power of the Reynolds number was about -2 and almost identical for all particle diameters studied (see regression equations in Fig. 5). This indicates that the particle Reynolds number affects the coefficient $C_{\text {Def }}$ in the same way for all particle diameters studied. Moreover, the larger deviations of particle settling velocity in a gas-liquid mixture from those in the single phase could be also caused by the increase in the value in the single liquid phase itself. For particles with diameter $5.5,11.0$ and $21.2 \mathrm{~mm}$, maximum $U_{\mathrm{p}}$ values were $0.3,2.6$ and $26 \%$ of the liquid velocity $U_{\mathrm{L}}$, respectively. Thus, fluctuations of relative particle to liquid velocity in gas-liquid flow affect more significantly the magnitude of the resulting liquid velocity as determined from Eqns (1) and (2) in the case of the largest $21.2-\mathrm{mm}$ particle.

\section{Effective buoyancy}

A proper definition of the buoyancy force acting on a tracer particle was also investigated. The effective settling velocity of the largest particle (diameter $21.2 \mathrm{~mm}$ ) was determined assuming that a particle experiences the gas-liquid dispersion density, $\rho_{\text {dis }} \doteq$ $\rho_{\mathrm{L}}\left(1-\varepsilon_{\mathrm{G}}\right)$. The calculated liquid velocities $\left(U_{\mathrm{Ldis}}\right)$ using such an effective particle velocity were much lower than the reference $U_{\text {Lref }}$ values owing to very high particle settling velocities (the error ranged from 16 to $54 \%$ ). It is evident that in the range of particle $(5.5-21.2 \mathrm{~mm}$ ) and bubble (around 3-6 $\mathrm{mm}$ ) diameters used, the Archimedes force based on the liquid density should be applied to determine the effective buoyancy force.

\section{Liquid velocity in an internal-loop ALR}

The precision of the magnetic particle-tracer technique was tested in an internal-loop ALR at different reactor configurations (variation of downcomer to riser cross-sectional area ratio $A_{\mathrm{D}} / A_{\mathrm{R}}$, height of draft tube $H_{\mathrm{DT}}$ and liquid level in separator zone $H_{\mathrm{L}}$ ). A magnetic particle with a diameter of $11.0 \mathrm{~mm}$ and low settling velocity $U_{\mathrm{p}}$ (up to $8 \mathrm{~mm} \mathrm{~s}^{-1}$ ) was used in the experiments. The method enables one to measure independently the particle velocity in both main reactor sections. Applying the continuity equation in the closed circulation loop of the ALR, a comparison between the liquid flow-rate in the riser and in the downcomer zones in an internal-loop ALR was carried out (Fig. 6). The particle settling velocity in the single-liquid phase $\left(U_{\mathrm{p}}\right)$ was applied as follows:

$$
Q_{\mathrm{LR}(\mathrm{D})}=A_{\mathrm{R}(\mathrm{D})}\left[V_{\mathrm{pR}(\mathrm{D})} \pm U_{\mathrm{p}}\right]\left(1-\varepsilon_{\mathrm{GR}(\mathrm{D})}\right)
$$

The values in Fig. 6 clearly demonstrate the satisfactory precision of the determination of the liquid velocity using the particle-tracer technique (the average error was 5.4\%). Application of the continuity equation in an airlift loop is also a simple, reliable way to verify the proper formulation of buoyancy acting on the tracer magnetic particle. The results confirmed the formulation of the buoyancy as a force based on the classical Archimedes force.

With regard to the practical use of the magnetic tracer method for ALR hydrodynamic measurements, magnetic particles with the smallest possible diameter and the lowest possible settling velocity should be used to minimize the experimental error. In this case, the changes in magnitude of the settling velocity due

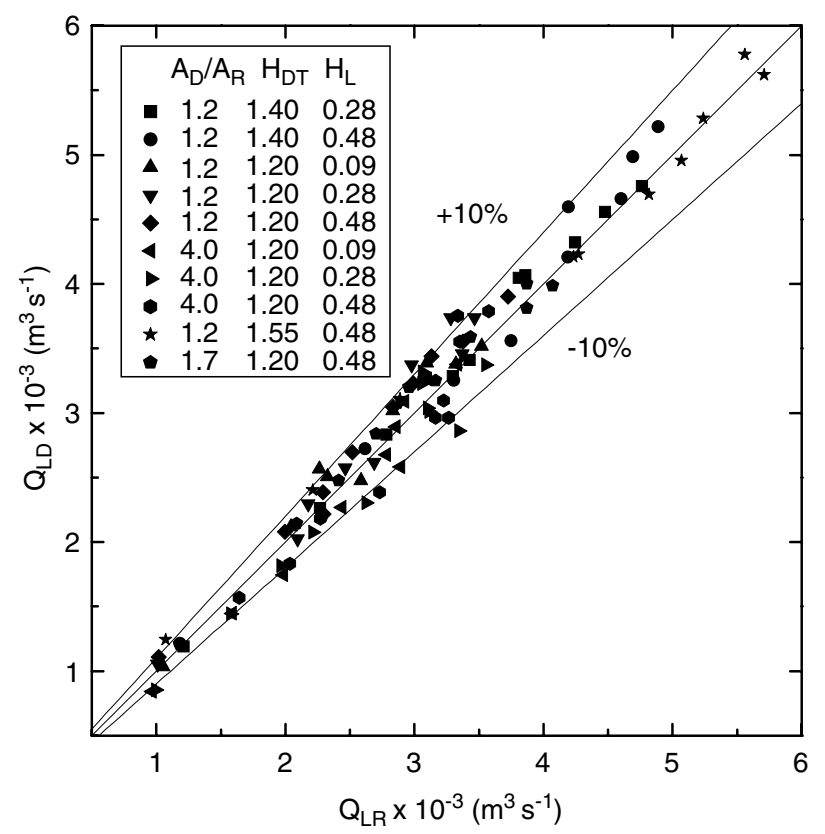

Figure 6. Comparison of the liquid flow-rate in the riser and downcomer zones in an internal-loop ALR at different reactor configurations based of the continuity equation. $A_{\mathrm{D}} / A_{\mathrm{R}}$ is the cross-sectional area ratio, $H_{\mathrm{DT}}$ the height of the inner draft tube (in $\mathrm{m}$ ) and $H_{\mathrm{L}}$ the height of liquid in the head reactor zone (in $\mathrm{m}$ ). 
to the particle-bubble interactions or fluctuations in fluid flow can normally be neglected in comparison with the magnitude of the liquid velocity commonly encountered in ALRs. In this case, values of the particle settling velocity in a single-liquid phase $\left(U_{\mathrm{p}}\right)$ can be applied for correction and an accurate determination of the liquid velocity. Moreover, if the tracer particle is used in non-Newtonian fermentation broths or high cell density systems, its settling velocity is strongly reduced owing to the high broth viscosity. The particle will then follow the fluid motion even more closely, giving more precise information on its velocity.

Studies dealing with the analysis of multiphase flow usually involve partial simplified models, which try to describe the motion of a tracer particle in bubble dispersion or a particle suspension under certain assumptions and simplified conditions. Of course, particle-bubble/particle interactions are much more complex in reality, therefore the adequate formulation of the interaction forces is still a contradictory topic in the literature. However, in recent years, much progress has been made by the involvement of rapidly developing CFD tools in solving sophisticated models, which should provide conclusive answers on interactions in multi-phase flow.

\section{CONCLUSIONS}

The application of the magnetic particle-tracer technique to the hydrodynamic study of the main sections of an ALR have been described. Unlike other tracer-following methods, the magnetic-detector coil technique is a low-cost, simple method, which allows the reliable measurement of the hydrodynamics in an internal/external-loop ALR during aseptic fermentation processes with an opaque broth.

A comparison of the measured liquid velocity using the magnetic-tracer method with reference values obtained by an independent flowmeter method showed good agreement, especially for smaller tracer particles with low free settling velocity, $U_{\mathrm{p}}$. The differences between the settling velocities in singleliquid and gas-liquid phases differ depending on the two-phase flow regime observed in the riser zone. This shows that diverse particle-bubble interaction mechanisms play a key role in different flow regimes. These discrepancies in settling velocity were analyzed using a $3 \mathrm{D}$ momentum transfer model. The model was able to predict satisfactorily the increase in the particle settling velocity in the homogeneous bubbly flow resulting from changes in the liquid velocity field due to bubble motion. However, it failed to predict the decrease in relative particle-liquid velocity in the churn-turbulent flow regime-emphasizing the importance of the inclusion of bubble-particle interactions. The experimental and theoretical data proved that an adequate definition of effective buoyancy acting on a tracer particle is the Archimedes force based on the liquid density. Comparison of independently determined liquid flow-rates in the riser and downcomer zones, based on the continuity equation applied to a closed circulation loop of an ALR, showed satisfactory accuracy of the magnetic particle-tracer method.

\section{ACKNOWLEDGEMENTS}

This research was supported by the FCT (Fundação para a Ciência e a Tecnologia), No. SFRH/BPD/5607/ 2001, awarded to Jaroslav Klein. The authors thank Associate Professors A Rodríguez and P Letón of the University of Alcalá de Henares, Spain, for their kind help and for providing the external-loop ALR used for reference measurements.

\section{REFERENCES}

1 Boyer C, Duquenne A-M and Wild G, Measuring techniques in gas-liquid and gas-liquid-solid reactors. Chem Eng Sci 57:3185-3215 (2002).

2 Larachi F, Chaouki J and Kennedy G, Three dimensional mapping of solids flow fields in multiphase reactors with RPT. AIChE f 41:439-443 (1995).

3 Chen J, Kemoun A, Al-Dahhan MH, Dudukovic MP, Lee DJ and Fan L-S, Comparative hydrodynamics study in bubble columns using computer-automated radioactive particle tracking (CARPT)/computed tomography (CT) and particle image velocimetry (PIV). Chem Eng Sci 54:2199-2207 (1999)

4 Kikuchi K, Takahashi H, Takeda $\mathrm{Y}$ and Sugawara F, Measurement of local residence time of particles in a draft-tube slurry bubble column using magnetic tracer particles. F Chem Eng fpn 28:483-486 (1995)

5 Wild G and Poncin S, Hydrodynamics of three-phase sparged reactors, in Three-phase Sparged Reactors, ed. by Nigam KDP and Schumpe A. Gordon and Breach, New York, pp. 11-113 (1996).

6 Fields PR and Slater NKH, Tracer dispersion in a laboratory air-lift reactor. Chem Eng Sci 38:647-653 (1983).

7 Philip J, Proctor JM, Niranjan K and Davidson JF, Gas holdup and liquid circulation in internal loop reactors containing highly viscous Newtonian and non-Newtonian liquids. Chem Eng Sci 45:651-664 (1990).

8 Kikuchi K, Takahashi H, Takeda Y and Sugawara F, Hydrodynamic behavior of single particles in a draft-tube bubble column. Can f Chem Eng 77:573-578 (1999).

9 Klein J, Blažej M, Godó S, Dolgoš O and Markoš J, Application of a magnetic tracer method for the characterisation of hydrodynamics in internal-loop airlift bioreactors. Chem Pap 54:456-466 (2000).

10 Dolgoš O, Klein J, Blažej M and Markoš J, Characterisation of hydrodynamics in airlift bio-reactor using the real fermentation broth. Presented at the 3rd European Congress of Chemical Engineering (ECCE3), Nuremberg (2001).

11 Klein J, Jurašcík M, Vicente AA and Teixeira JA, Continuous alcoholic fermentation in high cell density airlift bioreactor using flocculating yeast. Presented at the 30th International Conference of SSChE, Tatranské Matliare, Slovakia (2003).

12 Schmidt KG, Measuring techniques for evaluation of liquid velocity of gas-liquid systems. Verfahrenstechnik 17:593-597 (1983).

13 Gibilaro LG, Di Felice R, Waldram SP and Foscolo PU, Author's reply to comments by Clift, et al. Chem Eng Sci 42:194-196 (1987).

14 van der Wielen LAM, van Dam MHH and Luyben KCA, On the relative motion of a particle in a swarm of different particles. Chem Eng Sci 51:995-1008 (1996). 
15 Clift R, Seville JPK, Moore SC and Chavarie C, Comments on buoyancy in fluidized beds. Chem Eng Sci 42:191-194 (1987).

16 Fan L-S, Han LS and Brodkey RS, Comments on the buoyancy force on a particle in a fluidized suspension. Chem Eng Sci 42:1269-1271 (1987).

17 Grbavcic ZB and Vukovic DV, The effective buoyancy and drag on spheres in a water-fluidized bed. Chem Eng Sci 47:2120-2124 (1992).

18 Middleton JC, Measurement of circulation within large mixing vessels. Presented at the 3rd European Conference on Mixing (1979).

19 Jean R-H and Fan L-S, On the particle terminal velocity in a gas-liquid medium with liquid as the continuous phase. Can f Chem Eng 65:881-886 (1987).

20 Smuk D and Scott DS, Settling velocities of particles in bubbly gas-liquid mixtures. Can $\mathcal{F}$ Chem Eng 69:1382-1388 (1991).

21 Kundakovic L and Vunjak-Novakovic G, Mechanics of particle motion in three-phase flow. Chem Eng Sc. 50:3285-3295 (1995).

22 Kato Y, Nishiwaki A, Fukada T and Tanaka S, The behaviour of suspended solid particles and liquid in bubble columns. f Chem En. Fpn 5:112-118 (1972).

23 Smith DN and Ruether JA, Dispersed solid dynamics in a slurry bubble column. Chem Eng Sci 40:741-754 (1985).
24 Mazumdar D and Guthrie RIL, On the reduction in translational drag forces in bubbly Newtonian liquids. Chem Eng Sci 41:2965-2967 (1986)

25 Douek RS, Livingston AG, Johansson AC and Hewitt GF, Hydrodynamics of an external-loop three-phase airlift (TPAL) reactor. Chem Eng Sci 49:3719-3737 (1994).

26 Klein J, Vicente AA and Teixeira JA, Hydrodynamic considerations on optimal design of a three-phase airlift bioreactor with high solid loading. f Chem Technol Biotechnol 78:935-944 (2003).

27 Merchuk JC, Ladwa N, Cameron A, Bulmer M and Pickett A, Concentric-tube airlift reactors: effects of geometrical design on performance. AIChE 7 40:1105-1117 (1994).

28 Merchuk JC, Contreras A, Garcia F and Molina E, Studies of mixing in a concentric tube airlift bioreactor with different spargers. Chem Eng Sci 53:709-719 (1998).

29 Heijnen JJ and van't Riet K, Mass transfer, mixing and heat transfer phenomena in low viscosity bubble column reactors. Chem Eng f 28:B21-B42 (1984).

30 Kikuchi R, Tsutsumi A and Yoshida K, Stochastic analysis of bubble and particle motions in a 2-D three-phase reactor. f Chem Eng fpn 30:202-209 (1997).

31 Clift R and Grace JR, Continuous bubbling and slugging, in Fluidization, ed. by Davidson JF, Clift R and Harrison D. Academic Press, London, pp. 73-132 (1985). 